\title{
Pipeline for Making Images of Gas Flows in Binary Stars
}

\author{
Mercedes T. Richards ${ }^{1}$, Elena Slobounov ${ }^{1,2}$, Marshall Conover ${ }^{1}$, \\ John Fisher ${ }^{1}$, and Alexander Cocking ${ }^{1}$ \\ ${ }^{1}$ Pennsylvania State University, Department of Astronomy \& Astrophysics, 525 Davey Lab, \\ University Park, PA 16802, USA \\ email: mtr@astro.psu.edu,mjc5336@psu.edu,jgf5013@psu.edu \\ ${ }^{2}$ Pennsylvania State University, Research Computing and Cyberinfrastructure, 224G \\ Computer Building, University Park, PA 16802, USA \\ email: ess3@psu.edu
}

\begin{abstract}
The data collection and data analysis pipeline for the study and imaging of interacting binaries is outlined. This process includes the systematic collection of time-resolved spectra of individual systems, data reduction including subtraction of the stellar spectra, application of tomography codes to reveal images of the gas flows in $2 \mathrm{D}$ and $3 \mathrm{D}$, comparison of the observed spectrum with synthetic spectra of the accretion disk and gas stream, and application of 3D visualization techniques.
\end{abstract}

Keywords. (stars:) binaries: eclipsing, circumstellar matter, image processing, stellar dynamics

\section{Introduction}

A multi-dimensional process has been used to study the evolutionary behavior of interacting binary stars, from Algols to nova-like systems to gamma ray binaries. The focus has been on the visualization of the gas flowing between and around the stars in these binaries since nearly all of these systems are unresolved.

The pipeline for making and interpreting the images includes (1) the systematic collection of time-resolved multiwavelength spectra of individual systems over time-spans of 1-2 weeks, and over multiple epochs. (2) The data reduction stage includes subtraction of the stellar spectra to isolate the spectrum of the non-photospheric gas (e.g., Richards 1993). The stellar spectra have been represented by model atmospheres calculations (e.g., Richards \& Albright 1999) or by a representative spectrum based on the observed spectra (e.g., Prinja et al. 2011). (3) Tomography codes were applied to create 2D and 3D images of the gas flows (e.g., Richards 2004; Richards, Sharova, \& Agafonov 2010). This stage requires the same wavelength dispersion for all spectra so they can be merged into a single file for processing with the tomography codes. (4) The 2D tomograms are displayed using matLAB. The 3D tomograms are created as discrete sets of $2 \mathrm{D}\left(V_{x}, V_{y}\right)$ images over a grid of $V_{z}$ velocities. Hence 3D visualization techniques have to be applied to display the image in true 3D format; the Visualization Toolkit (VTK) was the most effective tool in this case.

The SHELLSPEC spectral synthesis code (Budaj \& Richards 2004) was created to model the accretion structures, e.g., the disk and gas stream. (5) It was tested on a well-known accretion disk system, TT Hya (e.g., Budaj, Richards \& Miller 2005). The observed spectra were compared with synthetic spectra of the accretion disk and gas stream, and tomography was used to illustrate how the separate accretion structures can be isolated. Also, (6) hydrodynamic simulations (e.g., Richards \& Ratliff 1998) can be performed to examine the suitability of the models and images derived from the observations. 


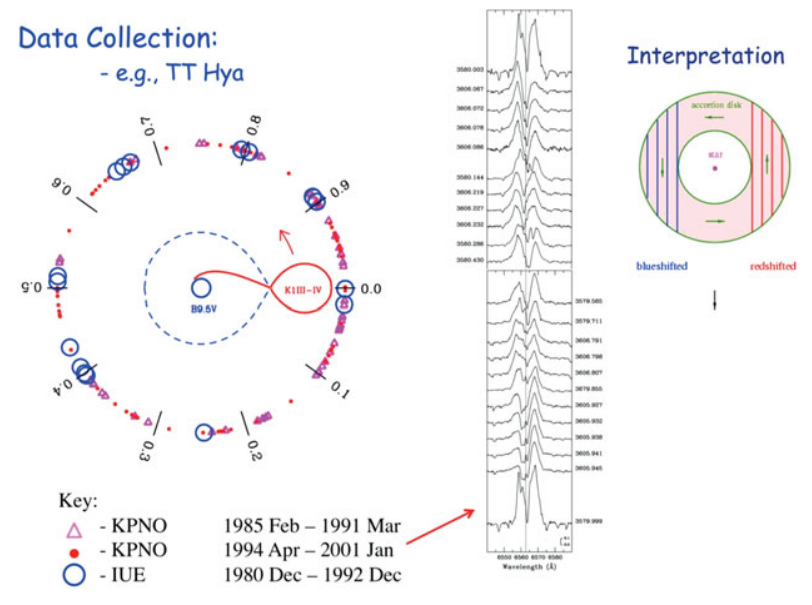

Figure 1. Data collection procedure illustrated in the case of TT Hya (Miller et al. 2007).

Data Reduction and Analysis

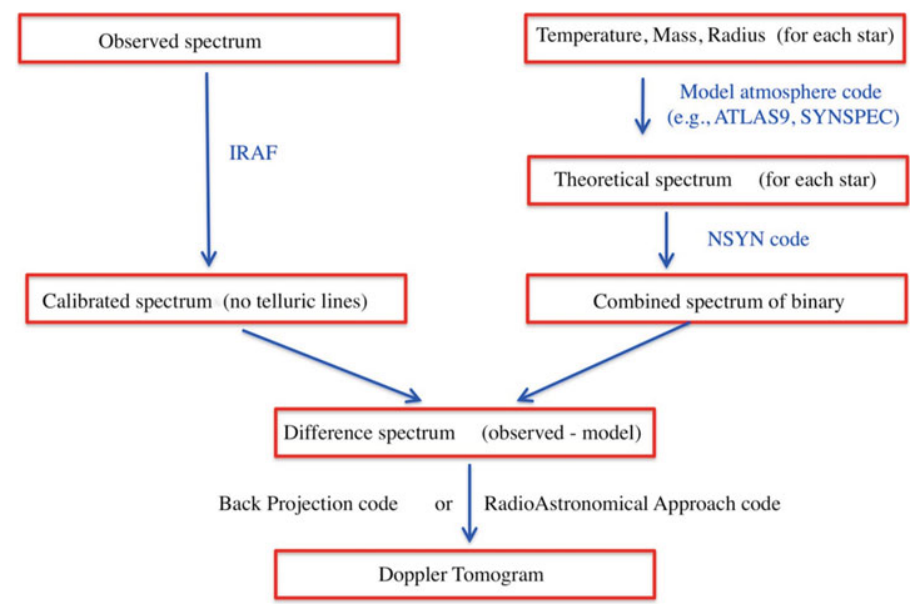

Figure 2. Data processing pipeline.

\section{Acknowledgements}

This research was supported by NSF grant AST-0908440 and an award from the Slovak Fulbright Commission and the Council for International Exchange of Scholars (CIES).

\section{References}

Budaj, J. \& Richards, M. T. 2004, Contrib. Astron. Obs. Skalnaté Pleso, 34, 167

Budaj, J., Richards, M. T., \& Miller, B. 2005, ApJ, 623, 411

Miller, B., Budaj, J., Richards, M. T., Koubský, P., \& Peters, G. J. 2007, ApJ, 656, 1075

Prinja, R. K., Long, K. S. Long, Richards, M. T., Witherick, D. K., \& Peck, L. W. 2011, MNRAS, in press

Richards, M. T. 1993, ApJ. Suppl., 86, 255

Richards, M. T. 2004, AN, 325, 229

Richards, M. T. \& Albright, G. E. 1999, ApJ. Suppl., 123, 537

Richards, M. T. \& Ratliff, M. A. 1998, ApJ, 493, 326

Richards, M. T., Sharova, O., \& Agafonov, M. 2010, ApJ, 720, 996 